\title{
Attention Explains the Transition to Unlimited Associative Learning Better Than Consciousness
}

\section{Carlos Montemayor}

Cognitive approaches to consciousness appeal to rational or epistemic traits in order to demarcate the boundary between conscious and non-conscious species. If a specific cluster of traits is present in a species, then it should qualify as conscious, given the importance of these traits for the kind of reflective and inferential capacities associated with conscious awareness in humans. A comparative and biologically informed cognitive approach affords the possibility of studying consciousness from a non-anthropocentric perspective because it appeals to traits found in other species that are not exclusive to human beings. It may also offer the best way of conceptualizing conscious awareness, an issue that notoriously resists empirical investigation, on the basis of scientific evidence on the nature and development of these traits. Birch, Ginsburg and Jablonka present in their important paper one of the most comprehensive and well-documented cognitive theories of consciousness. This alone is an impressive achievement, especially because the literature on consciousness is unfortunately riddled with verbal disputes and views that explicitly ignore the scientific evidence because, allegedly, all discussions about consciousness must be conducted introspectively or through a priori judgment. But this paper accomplishes much more, because it also serves as an example of how to integrate extensive scientific findings into a coherent and systematic scientific explanation of a particularly intractable topic. The authors deserve praise for enriching the literature on the science of consciousness with this interdisciplinary contribution.

Based on previous work by the authors, they bring insights from evolutionary biology, information theory, and animal cognition to the study of consciousness. Their key claim is that Unlimited Associative Learning (or UAL) serves as a distinctive set of traits that suffices to attribute consciousness to a species. According to Birch et al., "At the heart of the UAL approach is the concept of an evolutionary transition marker," defined in terms of a package of cognitive capacities concerning UAL, which jointly suffice for grounding phenomenal consciousness. These are abilities for cognitively processing compound stimuli and novel stimuli, as well as learning through second-order conditioning, trace conditioning, and flexible, easily rewritable associations with value (p. 11). The authors claim that these capacities constitute a natural cluster, and that they likely did not evolve independently from each other.

Given the nature of this cluster of capacities, a central question is what possible relation these capacities might bear to other cognitive abilities that are not sufficient for consciousness, but that are nonetheless necessary for conscious awareness. This commentary focuses on the importance of attention skills in the development of UAL, and it explains why the centrality of attention in UAL presents a considerable difficulty for the UAL approach. Attentional abilities are not just developmentally related to UAL, but in fact, they are explanatory (or even constitutive) of UAL. The main problem is that all the types of attention involved in UAL can be dissociated from consciousness. This means that while attention skills for UAL might be necessary for consciousness, they are not sufficient because they can all occur unconsciously. Thus, the possibility that UAL 
capacities might not be sufficient for consciousness cannot be eliminated, thereby challenging the key claim of the UAL approach.

The consciousness and attention dissociation (or CAD) is both theoretically accepted and empirically demonstrated through multiple experiments, from the neuro-anatomical to the behavioural and cognitive levels (Kentridge, 2011; Montemayor and Haladjian, 2015). All theories of consciousness assume that conscious cognition depends on the prior and more fundamental processing of information by attention routines. The empirical evidence robustly and abundantly shows that most forms of attention, and certainly those relevant to UAL, can occur unconsciously. Although CAD is not entirely uncontroversial, it certainly receives very strong theoretical and empirical support. Therefore, assessing the merits of UAL in the light of this dissociation is crucial to justify the claim that UAL suffices for consciousness. Moreover, because of the empirically informed nature of the UAL approach, the dissociation between consciousness and attention must be addressed since consciousness and attention likely had different evolutionary paths (Haladjian and Montemayor, 2015).

Consequently, although the authors are right about all their claims concerning the epistemic and evolutionary importance of UAL, their main proposal that these capacities constitute phenomenal consciousness is unjustified. More specifically, all these capacities are best understood in terms of attention routines. Given the empirically confirmed dissociation between phenomenal consciousness and attention, it is problematic to claim, as the authors do, that these capacities suffice for consciousness. The distinction between phenomenal and access consciousness plays a critical role here. Even if one grants that the capacities for UAL suffice for a kind of consciousness that is very similar to human conscious rationality, they all seem to involve access consciousness, or accessible content for action, decision-making, and thought, all of which are defined functionally (Block, 1995), and can be conceptualized, therefore, in terms of attention. These attention routines for the capacities involved in UAL can all happen unconsciously in humans, and they can certainly happen unconsciously in other species. The key disambiguation is that this is phenomenally unconscious cognition, or cognition without a unique "what it is like" that accompanies experiencing the information involved in these attention routines as part of one's subjective awareness.

Cross-modal attention for UAL, without necessitating any specific phenomenal character, provides a much better explanation of all the evidence offered by the authors from the field of animal cognition. In fact, the best explanation of the evolutionary transition to UAL is based on attentional skills for navigation and predatory behaviour, rather than the intricate phenomenon of the first person perspective and phenomenal consciousness. For example, because of the importance of meta-cognitive reflective capacities, some kind of dependence on language seems to be part of "what it is like" for humans to be conscious. But no dependence on inner speech or language can be assumed in explaining any of the capacities for UAL in other species. Actually, all the examples provided by the authors concern attention, rather than phenomenal character. Their emphasis on accessibility confirms this, as well as the fact that UAL is functionally specified in all the tasks performed by animals. Therefore, the attentional capacities that signal the transition to the Cambrian (p. 18) need not be phenomenally conscious. Given that attention suffices to explain UAL, but attention does not suffice to explain phenomenal consciousness (as the evidence on 
unconscious attention shows) UAL need not suffice for consciousness-a difficulty the authors must address in order to defend their main hypothesis.

One may object that UAL constitutes an agential perspective or the point of view of an agent that accomplishes epistemic achievements. Surely this perspective could be considered as a minimal kind of conscious awareness. In particular, the meta-cognitive epistemic endorsement involved in, for instance, second-order conditioning, is certainly a mark of conscious awareness in humans. How could a species satisfy such an epistemic standard and yet fail to meet the conditions for conscious access to information. Conscious access at the agent-level seems to be necessary for this type of epistemic achievement. Since this essentially involves the perspective of an epistemic agent, then there must be a minimal sense of "what it is like" to be this agent.

In response, while personal-level access to information that satisfies an epistemic standard is, typically, phenomenally conscious in humans, it is not the case that it always is. On the contrary, much of our higher-level cognition occurs implicitly, beyond our phenomenally conscious grasp. The capacity to process information unconsciously or implicitly is part of what makes us quite adaptive and general in our intelligent behaviour, and this implicitness of our epistemic lives can also be justified on purely theoretical and conceptual grounds (Siegel, 2017), independently of CAD. So even if access were highly correlated with phenomenality in humans, this would not entail that UAL necessarily implies phenomenal consciousness. Moreover, integrated attention can meet epistemic standards without any specific aspect of "what it is like" to think or perceive, based exclusively on the epistemic virtues of attention (Fairweather and Montemayor, 2017). Even in humans, epistemic agency does not necessitate phenomenal consciousness. One can grant that UAL may involve some level of access consciousness, but this kind of cognition can be dissociated from phenomenological requirements, and access consciousness can be understood in terms of attention (Stoljar, 2019).

One could also object that even if CAD presents difficulties for the UAL account, the traits that are constitutive of UAL are still the best explanation of consciousness that we have available. An inference to the best explanatory theory, on the basis of the available evidence, justifies UAL as a view that should be preferred over other less thorough and well-supported theories of consciousness. The problem, however, is that without eliminating the possibility that UAL is a theory of integrated attention for access consciousness, UAL cannot be an explanation of qualia or phenomenal consciousness. So what needs to be shown is that UAL is not only necessary, but also sufficient for consciousness. The fact that attention accounts for UAL leaves wide open the possibility that UAL is necessary, but not sufficient for conscious awareness.

In sum, the implications of CAD for UAL challenge the claim that the evolutionary transition to UAL signals the emergence of phenomenal consciousness. One can grant that UAL is a major transition in the evolution of cognitive capacities, and that it constitutes a cognitively integrated kind of epistemic perspective. But there are reasons for questioning that this marker is sufficient for phenomenal consciousness, chiefly the dissociation between consciousness and attention, which can be defended on conceptual and empirical grounds. The evidence, however, justifies what might be a substantial advantage in clarifying the status of UAL, since it could be a necessary but 
insufficient condition for phenomenal consciousness precisely because attention is necessary but insufficient for consciousness. This would be progress in the debate on the nature of consciousness.

In spite of this criticism, I am deeply sympathetic to the kind of approach Birch et al. offer. Although an extra informative component might be needed to explain conscious awareness, UAL provides a key marker towards systems that can sustain consciousness through integrated attention. This is a very welcome development in a debate that ranges from panpsychism to views that restrict consciousness only to humans. CAD presents challenges that the authors must address, but I am confident that looking for a marker like UAL is a step in the right direction, towards making the debate on phenomenal consciousness more scientifically rigorous and less anthropocentric.

\section{References}

Block, N. (1995). On a Confusion About a Function of Consciousness. Behavioral and Brain Sciences, 18(2): 227-247.

Fairweather, A. and Montemayor, C. (2017). Knowledge, Dexterity, and Attention: A Theory of Epistemic Agency. New York, NY: Cambridge University Press.

Haladjian, H. H. and Montemayor, C. (2015). On the Evolution of Conscious Attention. Psychonomic Bulletin and Review, 22(3): 595-613.

Kentridge, R. (2011). Attention without Awareness: A Brief Review. In Attention: Philosophical and Psychological Essays, eds. C. Mole, D. Smithies, and W. Wu, New York, NY: Oxford University Press: pp. 228-246.

Montemayor, C. and Haladjian, H. H. (2015). Consciousness, Attention, and Conscious Attention. Cambridge, MA: MIT Press.

Siegel, S. (2017). The Rationality of Perception. Oxford University Press.

Stoljar, D. (2019). In Praise of Poise. In Blockheads! Essays on Ned Block's Philosophy of Mind and Consciousness, eds. A. Pautz and D. Stoljar, Cambridge, MA: MIT Press: 511-536. 